\title{
IMPROVED REAL TIME COMMUNICATION USING STAR ROUTING PROTOCOL IN MANETS INFLUENCED BY FUZZY LOGIC
}

\author{
C P V N J Mohan Rao ${ }^{1}$ and S. Pallam Shetty ${ }^{2}$ \\ ${ }^{1}$ Department of Computer Science and Engineering, \\ Avanthi Institute of Engineering and Technology, Narsipatnam, AP \\ ${ }^{2}$ Department of CS and SE, AUCE (A), \\ Andhra University, Visakhapatnam-530003 \\ 1'mohanrao_c@yahoo.com, 2drspsetty@gmail.com
}

\begin{abstract}
In Mobile Ad hoc Networks, routing is a challenging issue. Basically, the working routing protocols for these networks are classified in to three categories like the reactive protocols, proactive protocols and the hybrid t protocols which were the combination of above two types of protocols. Among them proactive routing protocols category is selected for the present study. In proactive routing protocols, STAR with LORA approach is chosen because it has less control overhead when compared to ORA approach and other proactive routing protocols. It works on few defacto parameter values in a dynamic MANET environment. These static values for parameters are not suitable in a dynamic environment. With reference to the IETF draft, it is a time series problem. In this paper, an effort has been made to incorporate the soft computing technique, fuzzy logic based STAR to enhance the performance of a MANET to support real time communication. The proposed Fuzzy logic approach based STAR performance is evaluated using simulation through QualNet simulator. From the results, it is observed that the Fuzzy logic based STAR provides superior performance than the defactoSTAR protocol. The comparative performance was measured using the performance metrics End-to-end delay, Jitter and throughput. A number of simulation scenarios were executed for small, medium and large size networks. From the simulation results, we conclude that Fuzzy Logic based STAR outperforms for small, medium size networks.
\end{abstract}

Keywords - MANET, STAR, LORA, ORA, Fuzzy logic, Time to Live (TTL)

\section{INTRODUCTION}

In MANETs, performance is sensitive to quality, quantifiability and traffic load etc [1]. Examining completely different routing protocols performance in dynamic surroundings plays a significant role in economical routing. Mobile AdHoc networks are the wireless networks that were largely employed in the recent times within the real time world applications [2, 3, 4]. The nodes within the mobile networks are changing from time to time with relevance their position of the nodes within the actual design of the network. Because the nodes positions changes, the performance of the network influences plenty and therefore the impact was followed to the opposite nodes within the same network and other nodes that were connected to an equivalent networks[5, 6, 7]. The circumstantial networks are the self-configuring networks which is able to amendment the positions of

Received: July 5, 2019

Reviewed: October 1, 2019

Accepted: October 6, 2019

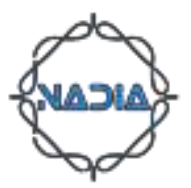


the networks and their locations supported the necessities of the network and their performance $[8,9,10]$. The mobile devices are allowed freely to maneuver chaotically and consolidate themselves subjectively [14, 15, 16,17].

The main drawback of these proactive protocols is the overhead with the maintenance of routes and the very frequent updating of routes such that to handle the node mobility and also for the utilization of more bandwidth than the usual amount of bandwidth. Among them STAR routing protocol is selected as it reduces the routing overhead with Least overhead routing approach (LORA) $[11,12,13]$.

According to IETF draft, fixed values do not set to be appropriate for dynamic environment $[22,23,24]$. From simulation results it is observed that, among the several defacto parameters Time to Live is found as the most significant parameter from Taguchi Design of Experiments $[25,26]$. The defacto value of Time to Live (TTL) is 6. It is a time sensitive parameter [27]. This can be solved by soft computing technique. Fuzzy Inference engine is able to fulfill these needs and helps in achieving a solution in finding optimized dynamic value for TTL $[18,19,20]$.

\section{REVIEW OF RELATED WORK}

Mohan Rao et al. [2] estimated the impact of TTL on STAR routing protocol and identified TTL as the most significant parameter influencing the performance of STAR.

Attada Venkataramana et al. [3] improved the QoS performance of DYMO routing protocol in MANETs by proposed Fuzzy based NTT DYMO. Optimal values of NTT are generated by the Fuzzy and used in the protocol configuration. The results reveal that FBNTT- DYMO outperforms traditional DYMO.

V Sireesha et al. [4] proposed and evaluated Fuzzy logic approach based OLSR performance by simulation using OPNET Modeler. The author observed that the Fuzzy logic based OLSR provides superior performance than the existing OLSR protocol. .

K. Narasimha Raju et al. [5] designed and analyzed the Fuzzy based Node Traversal Time AODV for improving QoS in MANETs..The author indicates that the result of AODV performance is enhanced by using the proposed FBNTTAODV.

Devi et. al., [6] described the fuzzy concepts used for routing in AdHoc networks.

Ayyasamy et. al., [7], presented fuzzy based QoS aware routing in MANETS. Chakrabarti et al., [8], discussed several challenges in providing QoS in MANETs.

\section{STAR ROUTING PROTOCOL}

In STAR routing protocol, every router informs the nature of the each link and node for the neighbors to reach the end source or the destination. The number of links being used by router in the favored pathway is called the router's source tree [14, 15]. A router notices its neighboring connections and aggregation of the neighboring connections of a router. The router creates its own source tree using the topology graph [16, 17]. The ORA or LORA approach can be used to update routing data depending on the bandwidth available in an ad hoc network. STAR promotes both. Under ORA, when source trees change, updates are sent [21]. 


\title{
4. DESIGN AND IMPLEMENTATION OF FUZZY BASED STAR ROUTING PROTOCOL
}

STAR considers the defacto parameter values suggested in the IETF draft [10]. The proposed method concentrates on the Time to Live (TTL) value which can be used for identifying and calculating the dynamic Time to Live value. According to the characteristics of a network routing protocol TTL plays a vital role. If TTL is too low, connection cannot be established and if it is too high energy consumption increases, which in turn affects the network life time. The defacto STAR suggests that the TTL value should be constant but it does not mention how this value should be adjusted with the network size and mobility speed. The proposed method "Fuzzy Based Time to Live (FB-TTL-STAR)" suggests that the TTL which can be considered as a perfect value for the size of the network and speed of the motion of the nodes in the network.

The following are the some of the default constants in the standard.

Time to Live $=6$

In FB-TTL-STAR, two inputs considered here are speed of the motion of nodes, size of the network and the output is taken as TTL.

Various numerical values considered for the fuzzification process in the current model are like the size of the network considered as 0 to 100 nodes, the speed of the network nodes considered as $0 \mathrm{~m} / \mathrm{sec}$ to $8 \mathrm{~m} / \mathrm{sec}$. The fuzzy association rules are generated through fuzzy rule editor. The expected output generated from the current model is from the fuzzy modeled system as the inputs are given on the basis of several fuzzy based rules and inputs. The actual output is expected or received from the reverse fuzzy process or going in next mode of the fuzzy process further.

Various fuzzy steps and other processes are used for the working of the fuzzy system and the utilization of fuzzy input values to non-fuzzy values. It is also required for the mapping of both non-fuzzy and fuzzy values such that the actual expected output values can be expected from the current model. Several performance metrics are used to study the quality of service being delivered to the end users. Some of those performance metrics are like the Jitter, throughput of the nodes and end to end delay. In order to implement the same, the most important factor to be found for the conduction of experiments and in the current model, the TTL had chosen as the most significant and important factor among them. The performance of FB-TTL-STAR with different TTL fuzzy values for different network sizes $(26,52,78)$ and mobility speeds $(2,4,6) \mathrm{m} / \mathrm{sec}$ is evaluated. At last, the performance of the current model is being compared with the other models and the results are tabulated and present in the results section. The comparison had been made in between the two models viz., FB-TTL-STAR model and the defacto STAR.

\subsection{FUZZY BASED STAR ALGORITHM FOR SIMULATION:}

\author{
// Algorithm for calculation of dynamic TTL for STAR using fuzzy logic \\ // input1 : Network size (nw) \\ // input2: Mobility speed(ms) \\ // output : Time to Live (ttl) \\ 1. Start \\ 2. Set Fuzzy inference system $\leftarrow$ Sugeno; \\ 3. Set inputl Name $\leftarrow n w$; \\ 4. Set range of input $1 \leftarrow[0,100]$; \\ 5. Set number of Membership function for input $1 \leftarrow 3$;
}


6. Set type of Membership function for input $1 \leftarrow$ triangular;

7. Set input2 Name $\leftarrow$ ms;

8. Set range of Input $2 \leftarrow[0,8]$;

9. Set the number of Membership function for input $2 \leftarrow 3$;

10. Set type of Membership function for input $2 \leftarrow$ triangular;

11. Set output Name $\leftarrow t t l$;

12. Set range of output $\leftarrow[0,1]$;

13. Set the number of the Membership function for output $\leftarrow 3$;

14. Set the type of Membership function for output $\leftarrow$ constant;

15. Write fuzzy if-then rules;

16. Set De-fuzzification method $\leftarrow$ wtaver;

17. Choose rule viewer for evaluation;

18. Record dynamic " $t$ ll" for the given inputs;

19. Select 3-D surface viewer;

20. Stop.

End procedure

Figure 1 show the architecture used in the model. Figure 2 shows membership function for the input variable network size and Figure 3 shows the membership function for the input variable Mobility speed. Table 1 shows fuzzy rule base for various combinations of inputs variables. Triangular shaped membership functions are preferred for output variable.

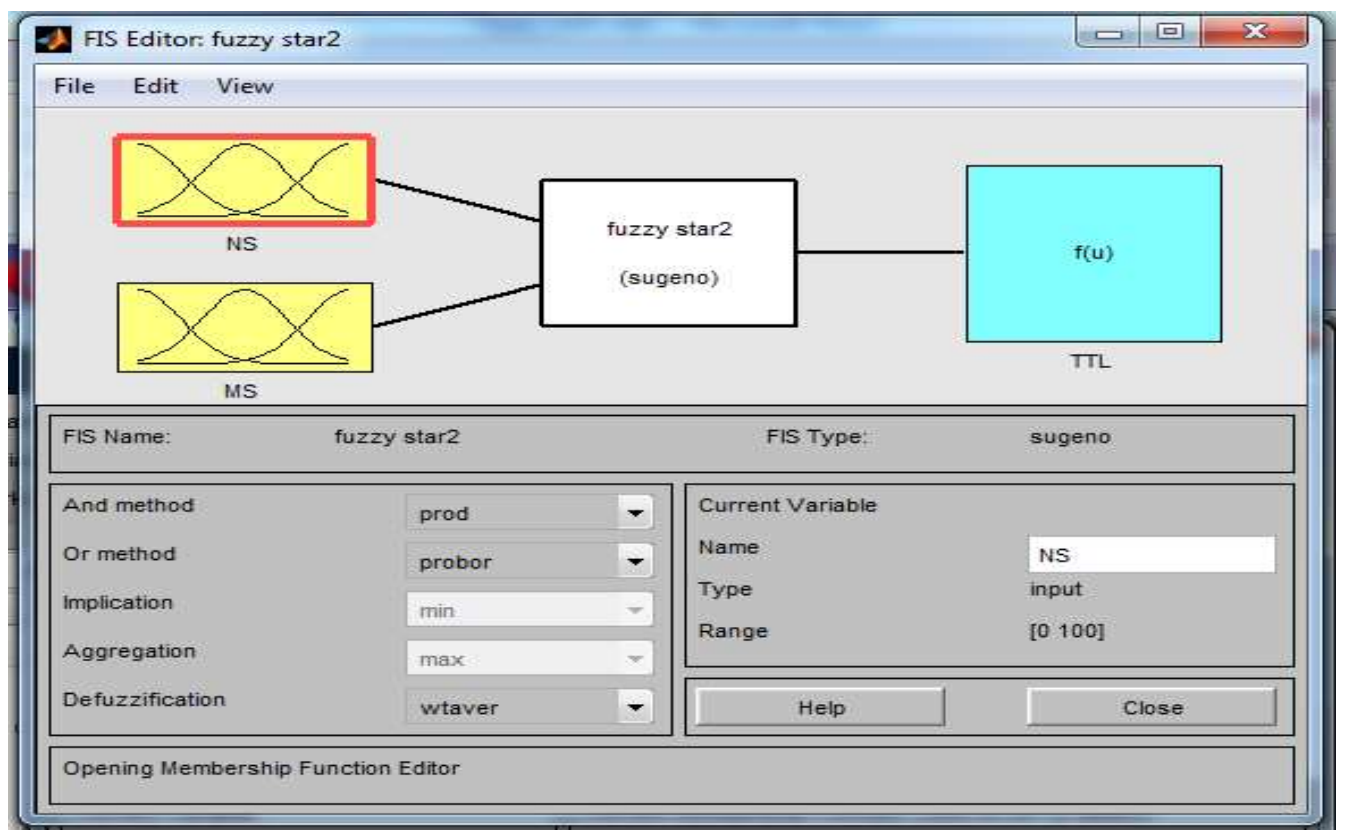

Fig. 1 Sugeno architecture of the FB-TTL-STAR 


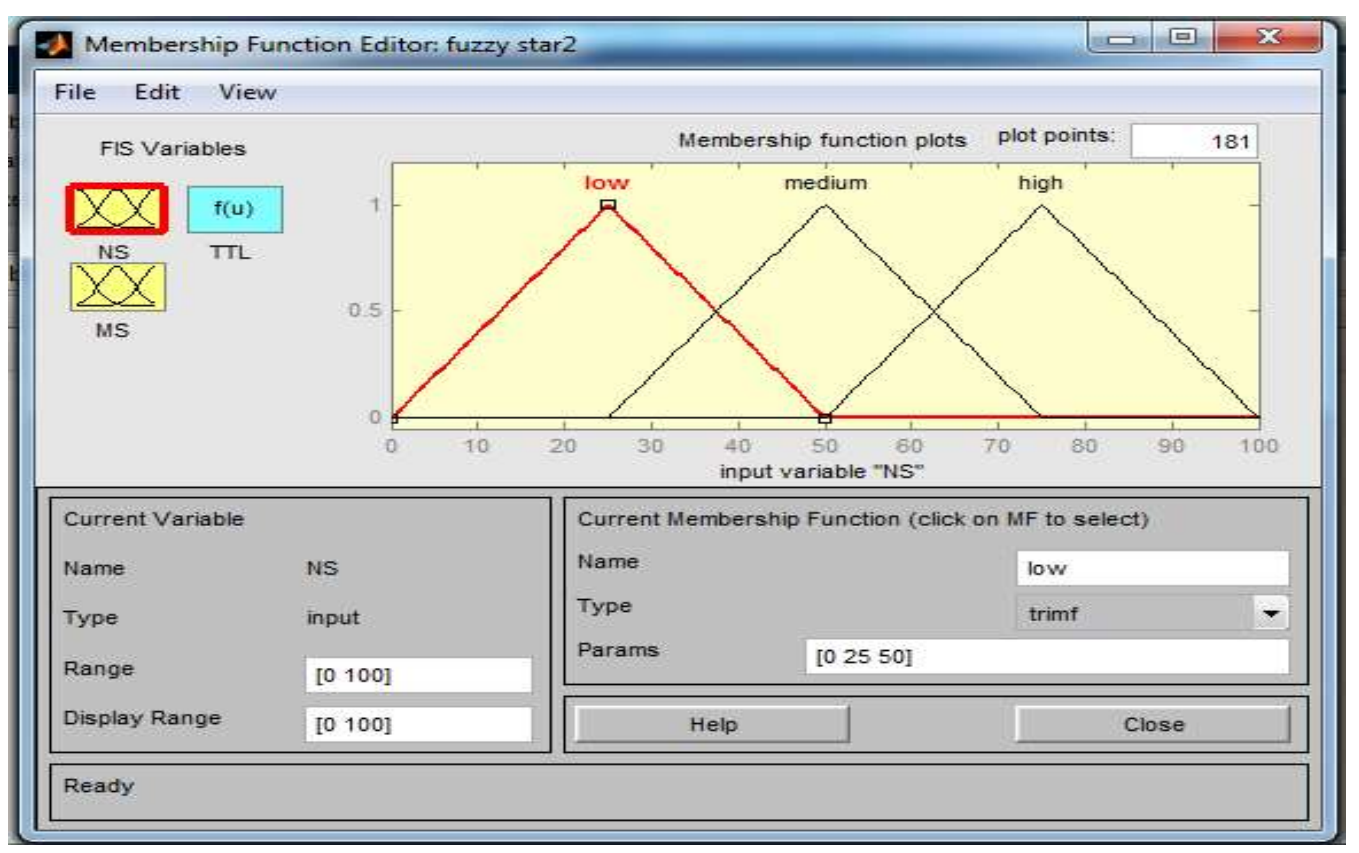

Fig. 2 Input variable network size with Membership function

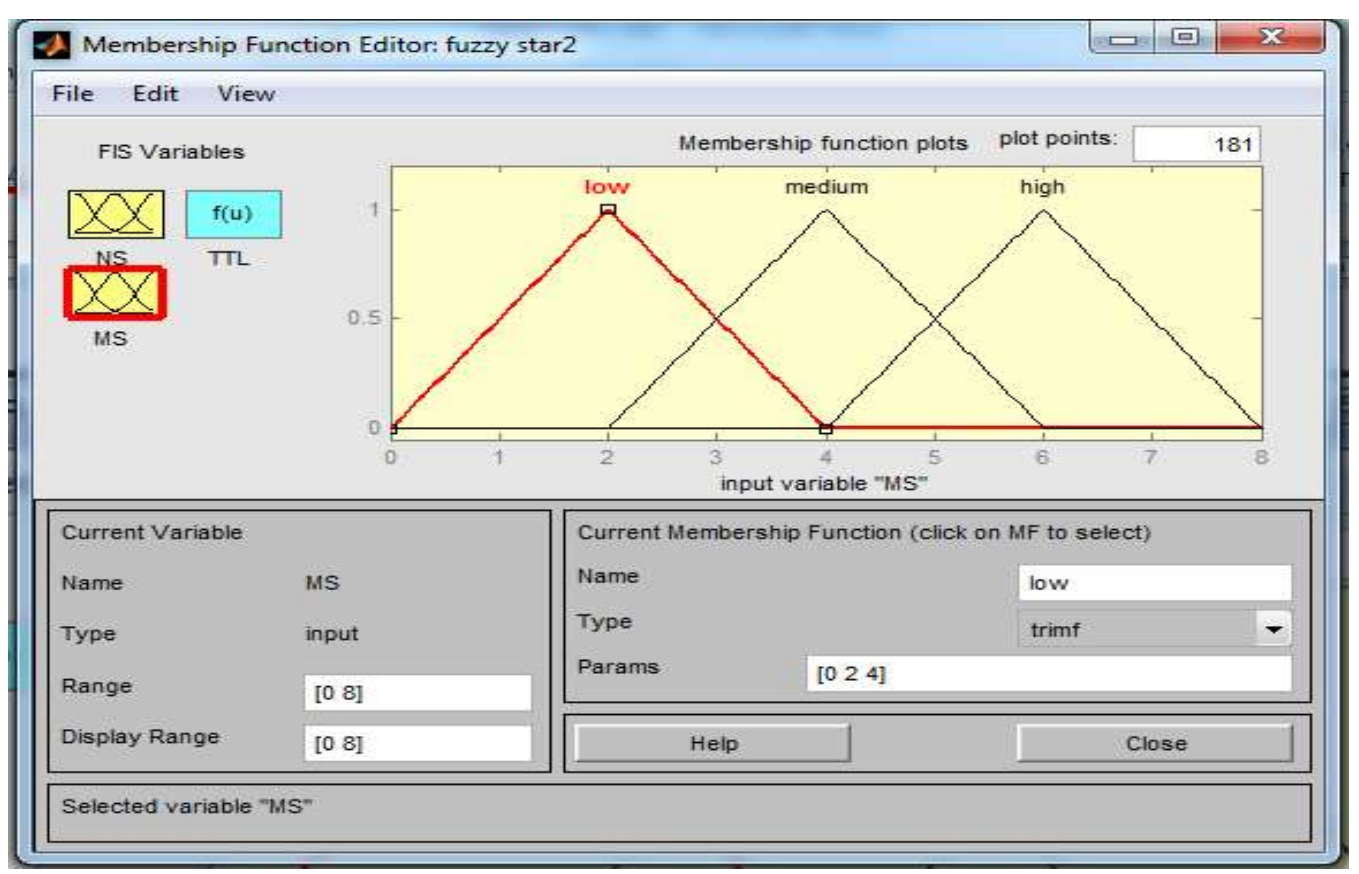

Fig. 3 Input variable mobility speed with Membership function 
Table I. Fuzzy Rule Base

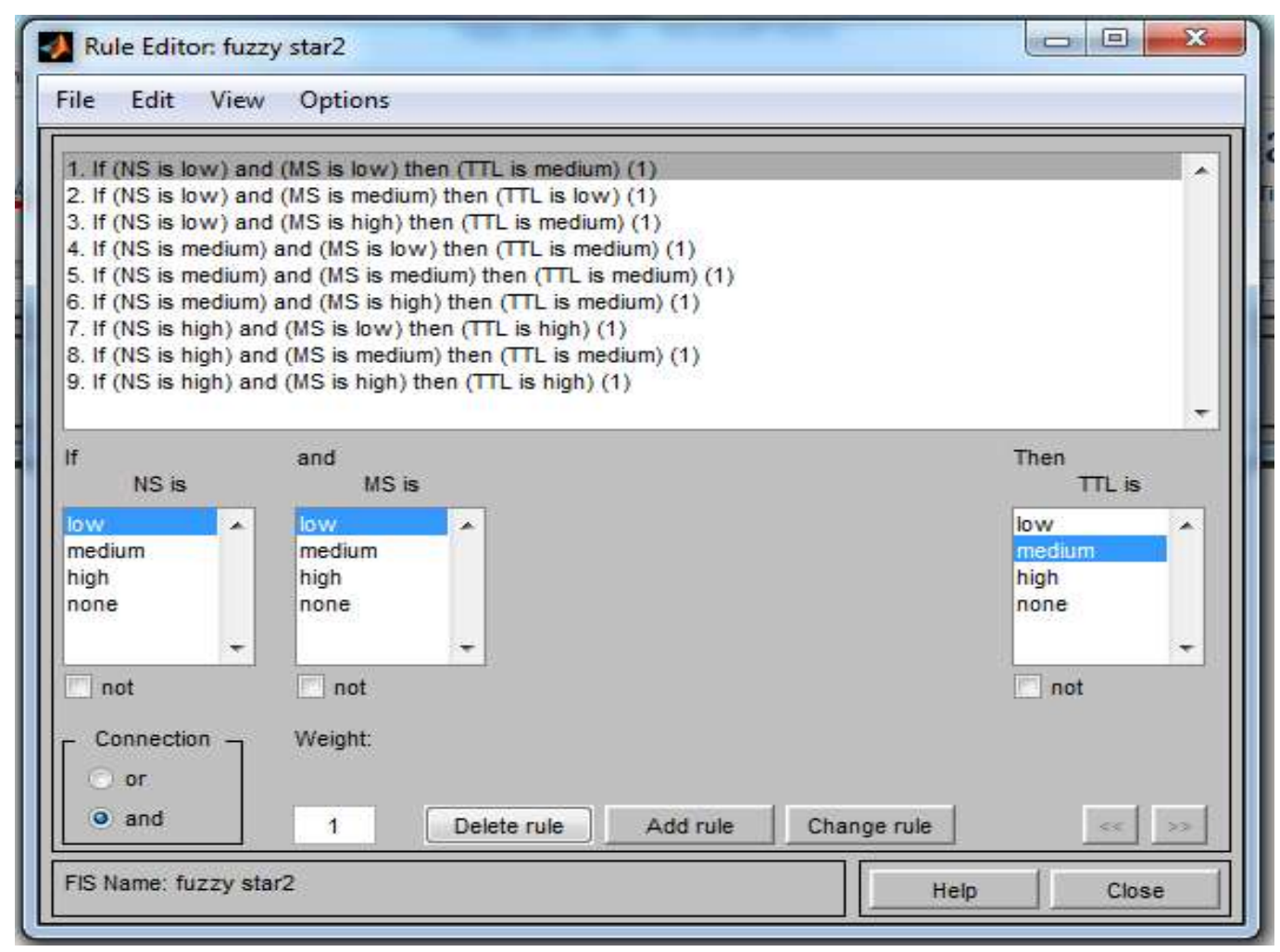

Figure 4 shows the Rule view for network size 50 nodes, mobility speed 4 \& output (TTL) 5 and Figure 5 shows Surface view with mobility speed, n nodes and output (TTL).

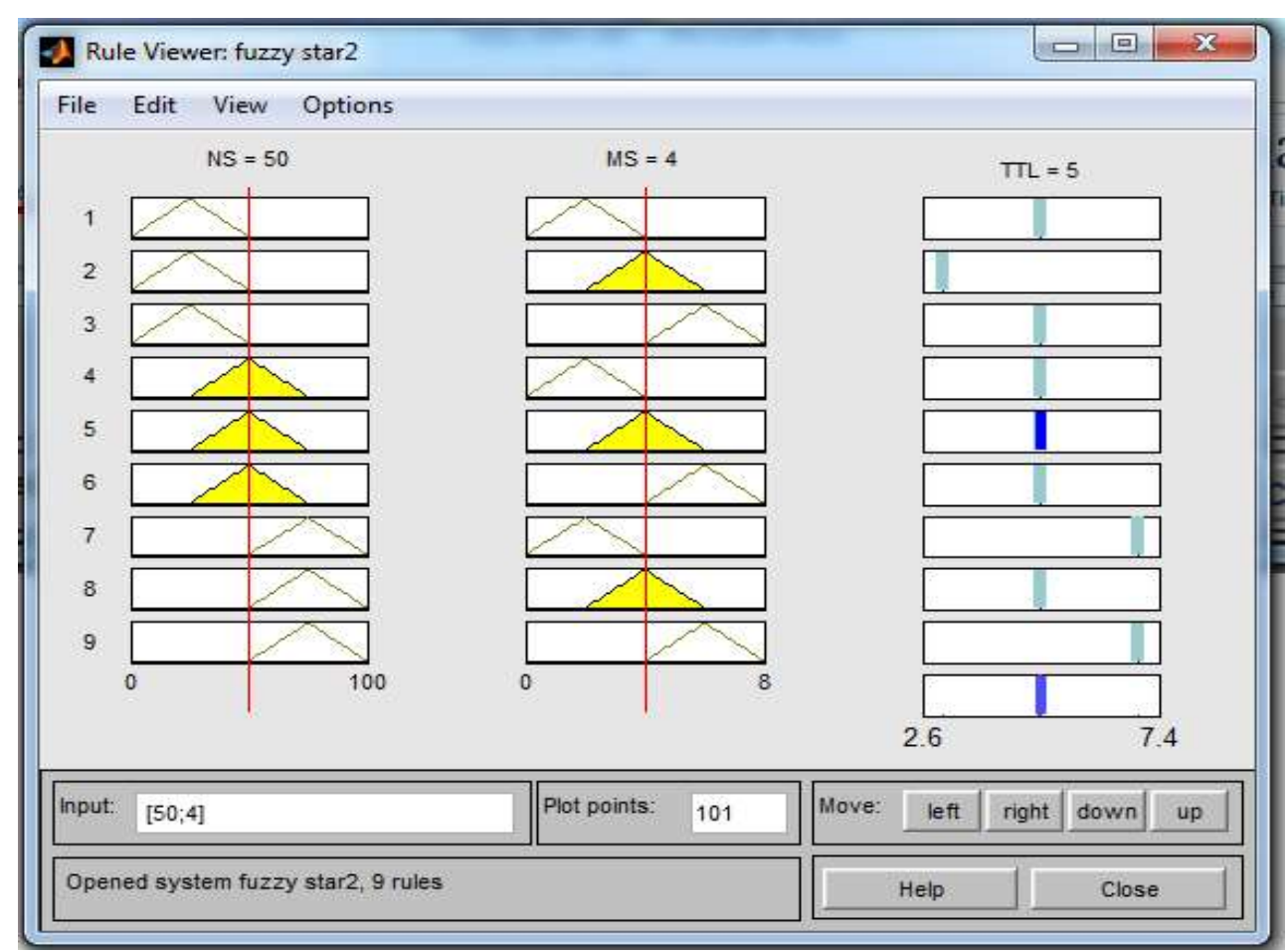

Fig. 4 Rule viewer for 50 nodes, mobility speed 4 \& output (TTL) 5 


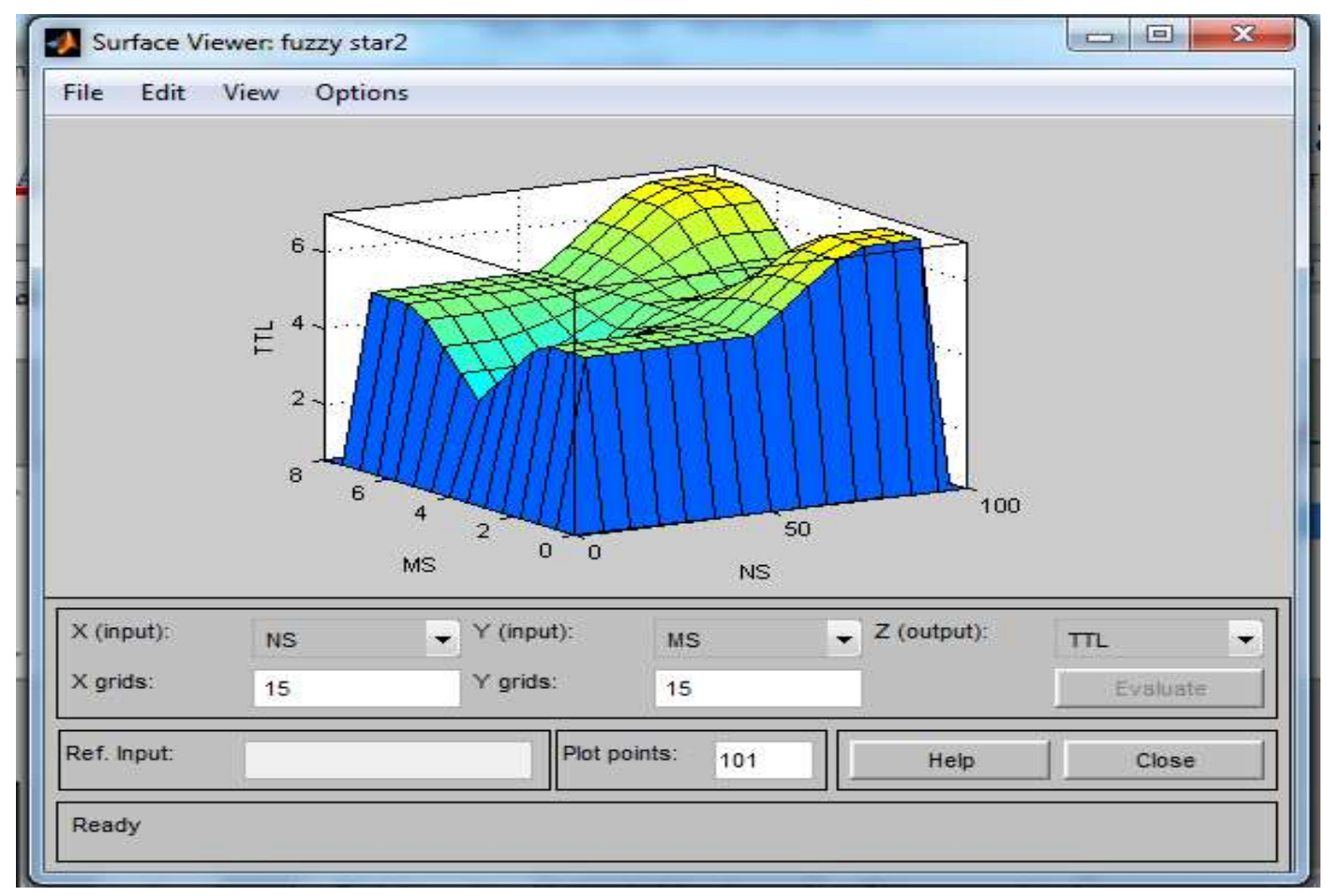

Fig. 5 Surface view showing mobility speed, network size and output (TTL)

\section{SIMULATION PROCESS}

Simulation setup considered for the conducting experiments is as follows. The simulator used for experimentation is QualNet Simulator. It is a tool for planning, testing, and training that simulates a real communication network's behavior. Researchers can evaluate a network's fundamental conduct and test network feature combinations that are supposed to work. The QualNet Network Simulation Software makes it possible for users to:

- Design new models of the protocol

- Optimize the existing and new models

- Use pre-configured or user-designed models to design big wired and wireless networks

- Analyze network performance and to optimize the same

The size of the network considered here with 26,52 and 78 nodes to identify or to observe the performance of the current model. The area to be made available for the simulation process is considered as the $1000 \times 1000$ square meters. The traffic type selection is done with CBR mode, the propagation model considered is two-ray ground model, random way point mobility is used and the speeds considered here are like the 2, 4 and $6 \mathrm{~m} / \mathrm{sec}$. The protocols used in the current model are the STAR and the FB-TTLSTAR protocols. The time considered for the simulation in the current model is around 300 seconds. Various considerations and numerical performance metrics considered in the current model are shown in the table as follows: 
Table II. Simulation Parameter Settings

\begin{tabular}{|l|l|}
\hline Routing Protocol & FB-TTL-STAR, STAR \\
\hline Time for the Simulation & $300 \mathrm{~s}$ \\
\hline Size of the Area & 1000 x1000 sqm. \\
\hline Model considered for Propagation & Two Ray \\
\hline Mode of Traffic & CBR \\
\hline Size of Packet & 512 bytes \\
\hline Number of Nodes & $26,52,78$ \\
\hline Type of Antenna & Omni directional \\
\hline Range for the Transmission & $250 \mathrm{~m}$ \\
\hline Range for the Receiver & $250 \mathrm{~m}$ \\
\hline Time taken for pausing the process & 0 sec \\
\hline Speed considered as maximum and minimum & $2,4,6 \mathrm{~m} / \mathrm{sec}$ \\
\hline buffered packets size & 100 \\
\hline Model considered for Mobility & Random Waypoint \\
\hline
\end{tabular}

\section{RESULTS AND ANALYSIS}

\section{Average Throughput:}

The throughput of the network model considered in the current model is as shown in the figure as follows,

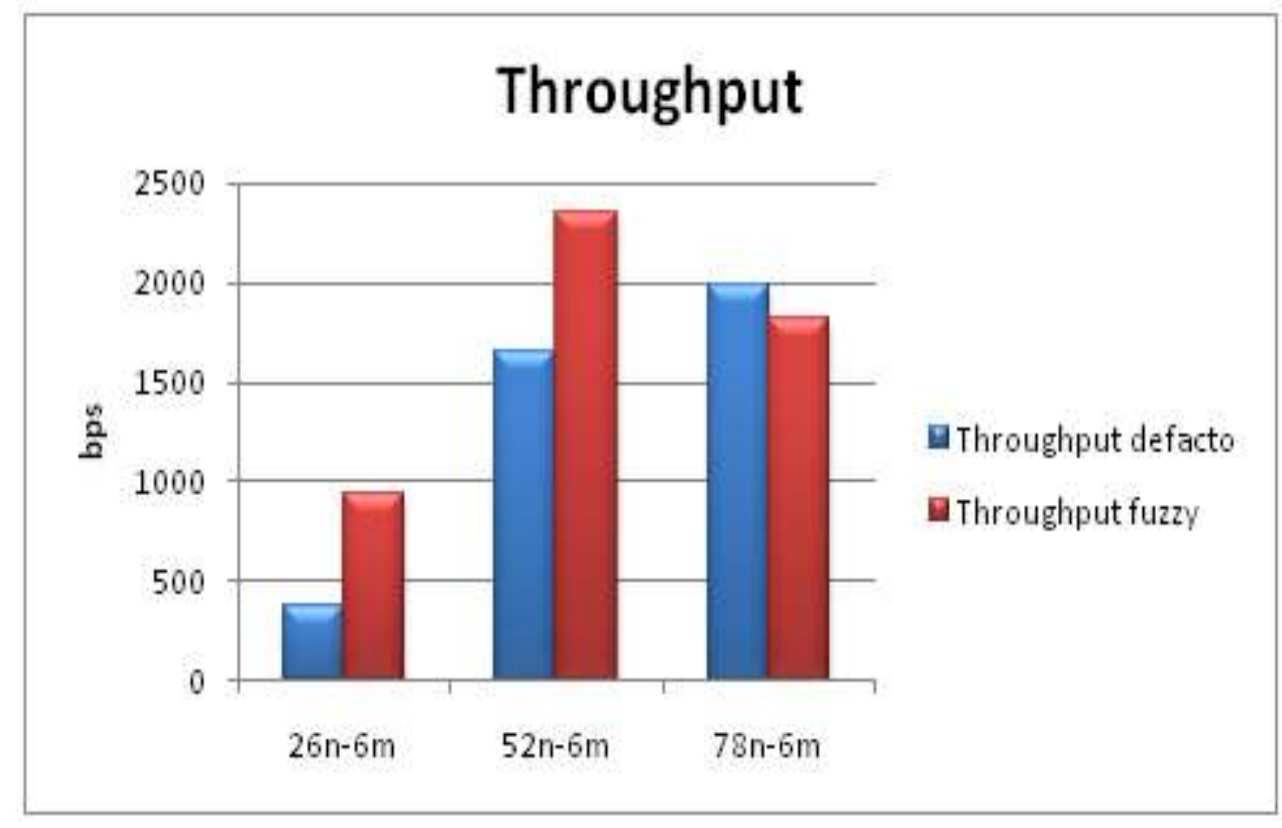

Fig. 6 Variation of Throughput with network size

From the graph in figure 6, it is observed that the Throughput for FB-TTL-STAR increases for small (26) and medium (52) network sizes but it is almost same for large(78) network size. 


\section{End-to-End Delay:}

This performance metric can be calculated as the time taken for a node to travel from one end of the network to the other end of the network. This time calculation is important to identify the number of packets to be sent at a point of time.

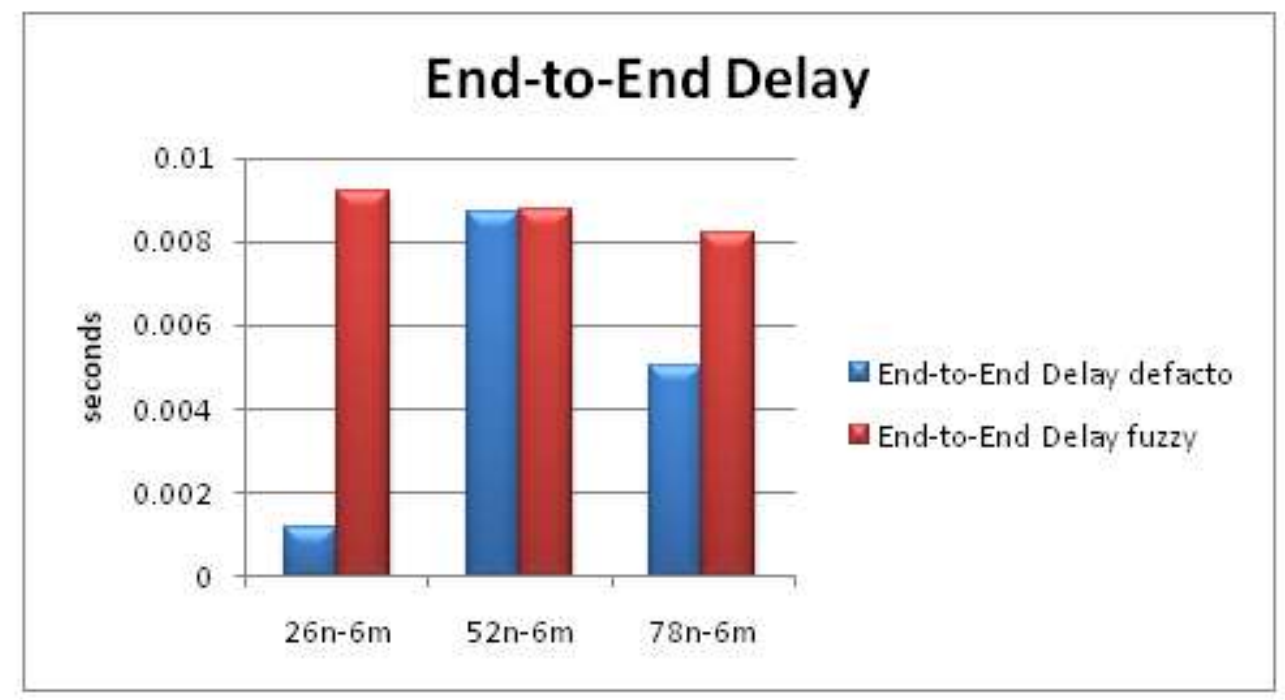

Fig. 7 The End to end delay to be observed for both defacto model and the fuzzy model

From the graph in Figure 7, the FB-TTL-STAR increases for all network sizes with respect to the performance measure End-to-End Delay.

\section{Jitter:}

From the graph in Figure 8, it is observed that the Jitter for FB-TTL-STAR decreases for small (26) and medium (52) network sizes but it is slightly increased at large (78) network size. Minimizing the jitter helps in enhancing the QoS in real time communications.

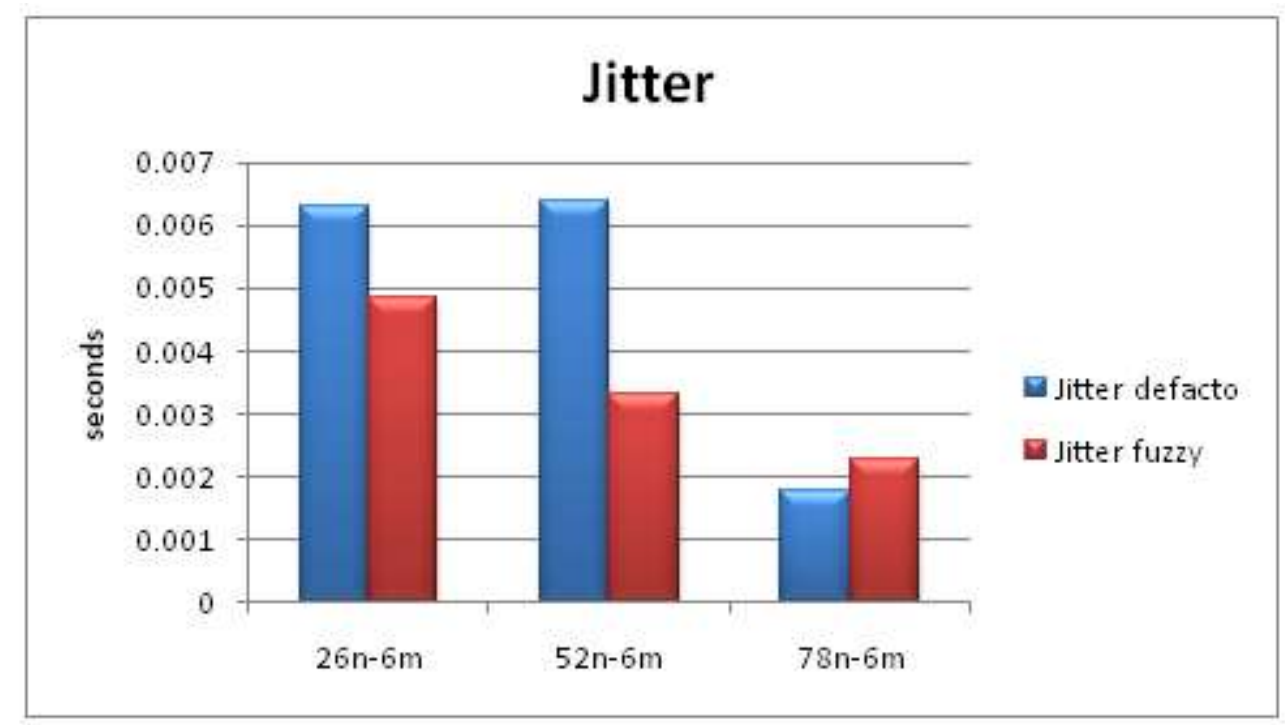

Fig. 8 Variation of Jitter with network size 


\section{CONCLUSIONS AND FUTURE SCOPE OF WORK}

A STAR protocol with TTL model and the combination of fuzzy logic are used for the design and to analyze the performance of a MANETs model such that to enhance the real time behaviour of the network models. This performance analysis can help and can increase the real time communication between the nodes in the networks. The experimentation is conducted for low, medium and high network sizes with 26, 52 and 78 nodes and mobility speeds of low, medium, high i.e., 2, 4, $6 \mathrm{~m} / \mathrm{sec}$ are used. Simulation scenarios are run on QualNet simulator. QoS metrics measured are Throughput, End to End Delay and Jitter.

By using Taguchi Design of Experiments, TTL is identified as the most significant factor. Using FB-TTL-STAR, TTL is fine-tuned from defacto value to dynamic value which enhances the performance of STAR routing protocol in real time communication. Therefore by using fuzzy logic concept, optimal values of TTL are obtained, thereby attaining the improved results. This paper presents that fine-tuning of significant parameter TTL values of the STAR routing protocol using MATLAB for improving the QoS performance in real time communication. After conducting the simulation model and as per the results obtained, it is noted that the FB-TTL-STAR performs superior than existing STAR for network sizes 26, 52 and mobility speeds 2, 4, $6 \mathrm{~m} / \mathrm{sec}$.

Based on the simulation results, it is noted that

- The throughput of the model for FB-TTL-STAR is enhanced by $60.5 \%$ for low network size, $29.82 \%$ for medium network size and it is decreased by 9.6\% for large network size when compared to defacto STAR.

- The End-to-End Delay of the model for FB-TTL-STAR is increased by $87.18 \%$ for low network size, $1.33 \%$ for medium network size and it is increased by $38.81 \%$ for large network size when compared to defacto STAR.

- Jitter for FB-TTL-STAR is decreased by $22.76 \%$ for low network size, decreased by $48.25 \%$ for medium network size and it is increased by $22.78 \%$ for large network size when compared to traditional STAR.

The work can be extended by using other soft computing techniques for further enhancement.

\section{REFERENCES}

[1] Venkataramana Attada and PallamSetty S (2017),"Enhance the Quality of Service in Mobile Ad Hoc Networks by Using Fuzzy Based NTT-DYMO", Wireless Personal Communication, doi:10.1007/s11277-017-3980-2.

[2] Mohan Rao C P V N J and S. Pallam Shetty, "DOE to identify the most significant factor to enhance the performance of defacto STAR for MANETs to support real time communication”, I-Manager's Journal on Wireless Communication Networks,Vol.8, No.1, 2019, Pp.9-16.

[3] Jin Wang, Jiayi Cao, R. Simon Sherratt, Jong Hyuk Park, "An improved ant colony optimization-based approach with mobile sink for wireless sensor networks", Journal of Supercomputing,Vol.74, No.12, Dec. 2018, Pp.6633-6645.

[4] Sireesha Vikkurty and S. Pallam Shetty," Design and Implementation of Fuzzy Logic Based OLSR to Enhance the Performance in Mobile Ad Hoc Networks", Springer Nature Singapore Pte Ltd - ICICCT 2019 - System Reliability, Quality Control, Safety, Maintenance and Management, 2020, Pp. 424-433.

[5] K. NarasimhaRaju, S.P. Setty, "Design and Analysis of Fuzzy based Node Traversal Time AODV for Improving the QoS in Mobile Ad Hoc Networks", International Journal of Computer Applications, November 2012,Vol.58, No.9, Pp.39-42.

[6] Devi, M., and Rhymend Uthariaraj, V., "Fuzzy based route recovery technique for mobile ad hoc networks", European Journal of Scientific Research, 2002, Vol.83, No.1, Pp.129-143.

[7] Ayyasamy, A., \& Venkatachalapathy, K., "Context aware adaptive fuzzy based QoS routing scheme for streaming services over MANETs", Wireless Networks, 2014, Vol.21, Pp.421-430.

[8] Chakrabarti, S., \& Mishra, A., "Quality of service challenges for wireless mobile ad hoc networks", Wiley Journal of Wireless Communications and Mobile Computing, 2004, Vol.4, Pp.129-153. 
[9] Peng Hai-yun, "Energy Consumption of Wireless Sensor Network Research Problem", International Journal of Internet of Things and Big Data, Vol. 1. No. 1. Dec. 2016.GVPress. Pp: 29-36.

[10] Jin Wang, Yu Gao, Kai Wang, Arun Kumar Sangaiah, Se-Jung Lim, “An Affinity Propagation based Self-Adaptive Clustering Method for Wireless Sensor Networks", Sensors, 2019, Vol.19, No.11, Pp.115.

[11] Nellore Harika, Muthyala Vamsilatha, Nakka Thirupathi Rao, Debnath Bhattacharyya and Tai-hoon Kim, "Performance Analysis and Implementation of Traffic Monitoring System using Wireless Sensor Network for reducing blockage in Traffic on Indian City Roads", Journal of Statistical Computing and Algorithm, Vol. 1. No. 1. Dec. 2017.GVPress. Pp: 15-26.

[12] Hyun-Soo Kim, Youngwan Cho, "A Design of Hovering System for Quadrotor UAV using MultiSensor Fusion", International Journal of Smart Device and Appliance, Vol. 4. No. 2. Dec. 2016.GVPress. Pp: 13-20.

[13] Yuyu Yin, Lu Chen, Yueshen Xu, Jian Wan, "Location-Aware Service Recommendation with Enhanced Probabilistic Matrix Factorization”, IEEE Access, 2018, Vol.6, Pp.62815-62825.

[14] Chong Min Hong, "Development of COP-based position sensor, International Journal of Smart Device and Appliance, Vol. 4. No. 2. Dec. 2016.GVPress. Pp: 29-34.

[15] Jin Wang, Yu Gao, Wei Liu, Arun Kumar Sangaiah, Hye-Jin Kim, “An Improved Routing Schema with Special Clustering using PSO Algorithm for Heterogeneous Wireless Sensor Network", Sensors, Feb. 2019, Vol.19, No.3, Pp.1-17.

[16] SiwooByun, "Efficient Transaction Control in Sensor Data Network", International Journal of Digital Contents and Applications for Smart Devices, Vol. 4. No. 2. Dec. 2017.GVPress. Pp: 1-6.

[17] Yuyu Yin, Song Aihua, Gao Min, Yueshen Xu, Wang Shuoping, "QoS Prediction for Web Service Recommendation with Network Location-Aware Neighbor Selection", International Journal of Software Engineering and Knowledge Engineering, 2016, Vol.26, No.4, Pp.611-632.

[18] Jia, Gangyong; Han, Guangjie; Jiang, Jinfang; Chan, Sammy; Liu, Yuxin. "Dynamic Cloud Resource Management for Efficient Media Applications in Mobile Computing Environments", Personal And Ubiquitous Computing, 2018, Vol.22, No.3, Pp.561-573.

[19] Hye Yun Kim, Seong Cheol Kim, Hyun Joo Park, "Priority and Delay Aware packet transmission MAC Protocol for Wireless Sensor Networks", International Journal of Security Technology for Smart Device, Vol. 5. No. 2. Oct. 2018.GVPress. Pp: 9-14.

[20] Jin Wang, Xiujian Gu, Wei Liu, Arun Kumar Sangaiah, Hye-Jin Kim, “An Empower Hamilton Loop based Data Collection Algorithm with Mobile Agent for WSNs", Human-Centric Computing and Information Sciences, Dec. 2019, Vol.9, No.18,. 10.1186/s13673-019-0179-4.

[21] Sengphil Hong, "Enhanced Re-route Filtering Scheme for Data Reporting in Wireless Sensor Networks", International Journal of Wireless and Mobile Communication for Industrial Systems, Vol. 3. No. 2. Oct. 2016.GVPress. Pp: 1-8.

[22] Nguyen Ngoc Minh, Myung Kyun Kim, 'CMP-MAC: A Cross-Layer, Multiple Pipeline-Forwarding MAC Protocol for Wireless Sensor Network", International Journal of Power Devices and Components for Smart Device, Vol. 3. No. 1. Dec. 2016.GVPress. Pp: 15-20.

[23] Ramesh Pusuluri, Reena Aggarwal, Shiva Chandar and Rajendra Kumar,"Pro-Active Routing Protocols in MANETs: A Comparative Study with Hybrid Routing Protocols", International Journal of Grid and Distributed Computing, November 2018, Vol.11, No.11, Pp. 29-38.

[24] Anmol Preet Kaur, Nitika Chowdhary and Jyoteesh Malhotra, "Performance Measure Modeling of Routing Protocols in MANET", International Journal of Future Generation Communication and Networking, December 2015, Vol. 8, No. 6, Pp. 215-228.

[25] Ajay Lala, Dr. Anand Bhaskar, and Dr. Prasun Chakrabarti, "Review on Energy Efficient Approaches on Dynamic Source Routing Protocol in MANET", International Journal of Smart Device and Appliance, Vol. 5. No. 1. Jun. 2017.GVPress. Pp: 1-10.

[26] Han Jidong, Gajendra Singh Dhakar, and Abhilash Sonker, "A Novel Approach to detect Denial of Service Attack in MANET", International Journal of Reliable Information and Assurance, Vol. 5. No. 1. Jun. 2017.GVPress. Pp: 21-30.

[27] Hyun Joo Park, Seong Cheol Kim, Hye Yun Kim, "Delay Aware Data Gathering Mechanism in Wireless Sensor Networks", International Journal of Security Technology for Smart Device, Vol. 5. No. 2. Oct. 2018.GVPress. Pp: 15-20. 
International Journal of Control and Automation Vol. 12, No. 10 (2019) 